Théologiques

Théologiques

\title{
Raconter la Russie des Juifs en 1839
}

\section{Mirela Saim}

Volume 15, numéro 1, 2007

Mémoires et usages religieux de l'espace

URI : https://id.erudit.org/iderudit/017643ar

DOI : https://doi.org/10.7202/017643ar

Aller au sommaire du numéro

\section{Éditeur(s)}

Faculté de théologie et de sciences des religions, Université de Montréal

\section{ISSN}

1188-7109 (imprimé)

1492-1413 (numérique)

Découvrir la revue

\section{Citer cet article}

Saim, M. (2007). Raconter la Russie des Juifs en 1839. Théologiques, 15(1), 113-129. https://doi.org/10.7202/017643ar

\section{Résumé de l'article}

L'article examine les mémoires des voyages en Russie de Max Lilienthal, un texte d'exception sur la Russie juive : le jeune voyageur juif venu d'Allemagne décrit l'image d'une communauté religieuse et ethnique à l'aune de la crise dramatique des Lumières, drame qui se déploie sur le territoire de la Russie tsariste. À la fois engagé et curieux de tout ce qui a trait à la situation des Juifs, Max Lilienthal construit dans ses lettres de Russie un espace idéologique particulier, à la fois cloisonné et divers, cartographiant la condition juive dans l'empire de Nicolas $\mathrm{I}^{\mathrm{er}}$ et imposant une comparaison éclairante avec le texte du voyage russe d'Astolphe de Custine, écrit à la même époque, mais beaucoup mieux connu.
Tous droits réservés @ Faculté de théologie et de sciences des religions, Université de Montréal, 2007
Ce document est protégé par la loi sur le droit d'auteur. L'utilisation des services d'Érudit (y compris la reproduction) est assujettie à sa politique d'utilisation que vous pouvez consulter en ligne.

https://apropos.erudit.org/fr/usagers/politique-dutilisation/ 


\title{
Raconter la Russie des Juifs en 1839
}

\author{
Mirela SAIM \\ Faculty of Religious Studies \\ McGill University, Montréal
}

«La ville de Saint-Pétersbourg dépasse tout! Cinq milles en circonférence et rien que des palaces! C'est vraiment unique! Qui n'a pas vu SaintPétersbourg n'a rien vu! Une autre ville pourrait, peut-être, avoir plus d'habitants, mais pas plus de grandeur ou de beauté1.» (Philipson 1915, 133) À peine débarqué en Russie, son regard posé sur l'espace d'une ville étrangère, un jeune homme exalté faisait part de son émerveillement dans une lettre adressée à sa famille, essayant de communiquer ses émotions par ces paroles. Et, dans une formule plus synthétique, il ajoutait: "Tout est ici colossal et indescriptible! » (136) Le jeune enthousiaste, maniant ainsi l'hyperbole, était un voyageur juif allemand rempli d'espoirs et de projets réformateurs pour «son peuple»: il s'appelait Max Lilienthal (1814-1882) et, en 1839, il arrivait en Russie, venant de Munich et de Leipzig, villes prussiennes de la Réforme.

Quinze ans plus tard, en 1854, il énonçait ainsi le but de son voyage de 1839: "quelque chose devait se faire pour les Juifs de la Russie qui, seuls parmi leurs coreligionnaires, étaient restés derrière la civilisation de notre âge» (159). Autrement dit, il s'agissait pour lui « de soulever les millions de Juifs à un niveau plus élevé » (ibid.). Comme on le voit, son voyage en Russie avait dès le début été placé sous le signe de la religion et du politique — la traversée de l'espace étant du départ constituée en histoire civilisatrice ! En fait, son aventure russe aurait même dépassé ses propos ambitieux et bien vagues, car avant son départ de Russie, en 1845, après avoir traversé l'espace juif deux fois, il aurait contribué au changement radical de la réglementation du statut institutionnel de la question juive sous le tsar Nicolas I $^{\text {er }}$ (1825-1855).

1. La traduction française des textes de Lilienthal est mienne.

(C) Revue Théologiques 2007. Tout droit réservé. 
Dans ce qui suit, je voudrais examiner le lettres ${ }^{2}$ de Lilienthal tenant lieu de ses mémoires sur ses voyages en Russie, ces textes exceptionnels sur la Russie juive ${ }^{3}$ laissés par un voyageur à la fois engagé et si curieux de tout ce qui a trait à la situation des Juifs, à leur statut, à leurs coutumes et à leur identité religieuse et ethnique. Les mémoires du voyage de Lilienthal ont un caractère remarquable: ils font de la représentation de l'espace une question centrale du discours identitaire juif, discours à l'aune de la modernisation et de la confrontation avec la tradition religieuse et l'autorité politique. L'espace du mémorable devient ainsi l'espace du réformable.

Or, c'est justement la possibilité du progrès, ses limites et son imminence qui sont mises en question dans ces récits de voyage en Russie, qui engagent un discours tout à fait unique sur l'histoire, la tradition et l'espace.

L'espace russe est, avant la guerre de Crimée, perçu comme un immense espace culturel du retard, surtout politique, causé par le despotisme d'un système réactionnaire figé. L'empire du Tsar, immense et multinational, offrait aux voyageurs européens occidentaux la fascination d'un miroir renversé du devenir historique: si l'Amérique visitée par Alexis de Tocqueville (1805-1859) présente l'ébauche de l'avenir démocratique, la Russie, vue par Astolphe de Custine (1790-1857) et Lilienthal offre l'image trouble d'un obscurantisme catastrophique en quête de modernisation ${ }^{4}$.

\section{Les voyages en Russie, un emblème de la modernité ?}

Longtemps ignorée, souvent mal connue ou bien imaginaire, la Russie arrive à son moment de confrontation avec l'Occident seulement au début du XIX ${ }^{e}$ siècle : c'est pendant la première partie de ce siècle que les voyages

2. Ces lettres sont parues de 1845 à 1848 dans la presse juive allemande; puis, complémentairement, une version anglaise partielle du texte a été publiée de 1854 à 1855 dans la presse juive américaine (The Israelite, devenue The American Israelite). Finalement, l'entière collection des lettres sur la Russie a été publiée dans Max Lilienthal, American Rabbi: Life and Writings, édité par D. Philipson (1915). Ce dernier a ajouté des lettres inédites envoyées à la famille et écrites en allemand et en français.

3. Dans ce qui suit, l'expression «Juifs de Russie » réfère aux Juifs résidant sur le territoire de l'Empire russe après 1831, donc ceux de la Pologne aussi.

4. La carte du politique était à l'époque structurée selon un modèle à la fois chronologique et topographique qui faisait de l'Angleterre le centre libéral de l'idéologie sociale et politique, et de l'Espagne et de la Russie les périphéries continentales caractérisées par des systèmes autocratiques oppressifs à la fois extrêmes et arriérés; l'Amérique offrait une image iconique de l'avenir démocratique à la fois éloigné et ambigu. 
des intellectuels occidentaux en Russie ont reçu un cachet particulier, surtout grâce aux premiers travaux monographiques offrant une image plus détaillée et plus réaliste de ce territoire de l'immensité froide et éloignée. Les premiers géographes, ethnographes et anthropologues occidentaux donnent une représentation discursive de la Russie qui se propose d'expliquer et d'analyser "scientifiquement» l'immensité du territoire, tout en tenant compte de la grande variété culturelle de ses habitants. Dans ce discours d'analyse et de représentation, le politique et l'identitaire se partagent: le discours sur la Russie dans la première partie du XIX siècle semble dominé par le désir de comprendre le secret du retard politique russe. Dans ce siècle de la démocratie libérale, le système politique russe, marqué par un absolutisme extrême et constitué de despotisme et de cruauté, est vu comme survie anachronique du passé, d'un Ancien Régime qui échappe à la logique de l'histoire et à l'impératif du progrès.

Plusieurs événements de l'histoire du XIX ${ }^{\mathrm{e}}$ siècle ont d'ailleurs intensifié l'intérêt des auteurs occidentaux pour la Russie. La participation russe à la "Sainte-Alliance ", à côté de l'Angleterre, la Prusse et l'Autriche, et aux victoires antinapoléoniennes aboutissant à la conquête de Paris par les armées tsaristes, la conquête de la Pologne, les fréquents échanges des élites politiques et artistiques russes avec les Français et les Allemands sont tous des facteurs qui mènent à une intensification des liens et à une meilleure connaissance réciproque. Mais, en dépit de tout cela, la Russie restera pour longtemps et pour plusieurs un espace du mythe et de l'imaginaire. Car c'est aussi le siècle du romantisme, où l'imaginaire russe jouit d'une fascination renouvelée; en tant qu'objet discursif, la Russie s'impose comme objet de découverte et de connaissance.

Inévitablement, pour plusieurs, la Russie devient aussi un espace de questionnement et d'apprentissage, comme l'a bien remarqué le plus important voyageur en Russie de ce temps, le marquis Astolphe de Custine, qui écrivait à propos de son voyage en Russie: «en disant la vérité sur la Russie, je ferais une chose neuve et hardie. » (1990, vol. 1, 20)

Dans le siècle fortement marqué par l'apprentissage de la démocratie, la Russie du Tsar se pose d'emblée comme l'illustration même de l'envers du miroir, objet de connaissance nécessaire, surtout si l'on veut mieux apprendre les bienfaits d'un ordre politique encore en train de se faire, la démocratie moderne: "J'allais en Russie, avoue Custine, pour y chercher des arguments contre le gouvernement représentatif, j'en reviens partisan des constitutions. » (ibid.) 
Dans l'aventure du politique, qui est celle de la démocratie libérale moderne, l'Amérique décrite par Tocqueville comme un avenir inquiet et inquiétant, égalitaire, mal assuré et mal géré, reste contre-utopique et équivoque. Par contre, la Russie et l'Espagne, aux marges extrêmes de l'Europe "civilisée ", représentaient encore l'espace d'une géographie politique à la fois anachronique et liminaire, et, peut-on affirmer aussi, anachronique, car liminaire! Situées aux antipodes l'un de l'autre, en marge d'une Europe romantique et post-luministe, les espaces ibérique et russe sont du fait même de leur retard politique l'objet d'une littérature de voyage en quête d'un réel à la fois signifiant et symbolique dans l'ordre du progrès et de la modernité.

Or, voilà que, à la fin des années 1830 , deux récits de voyage nous révèlent une Russie toujours inexplorée et secrète. C'est la Russie des minorités religieuses, décrite dans les lettres semi-privées d'Astolphe de Custine, parues dans La Russie en 1839, et dans celles de Max Lilienthal, dans My Travels in Russia ${ }^{5}$. Les deux récits ont une inscription générique similaire, d'écriture épistolaire sérielle, et portent sur un contenu fortement chargé de rhétorique informé par une idéologie bien énoncée et orientée vers des choix politiques clairs et des options religieuses tout aussi claires.

Sans se connaître ou se rencontrer, les deux hommes ont voyagé en Russie presque en même temps et ont tous les deux donné des récits de voyage très critiques du régime tsariste ${ }^{6}$, tout en cherchant à comprendre l'esprit de l'immensité russe par des rencontres variées et par des conversations avec des représentants du régime très haut placés, en l'occurrence le tsar Nicolas I $^{\text {er }}$ et son ministre de l'Éducation et de "l'Amélioration civique ", S.S. Uvarov. À leur insu, dans des discours fort différents, les deux voyageurs auront consigné, dans leurs contrariantes et convergentes mémoires, les traces indélébiles et paradoxales du bonapartisme politique dans l'idéologie des élites russes, tout en discutant avec passion la place de la religion dans la culture de la modernité.

Mais ici, les coïncidences cessent et les différences commencent: Custine voyage en Russie pour comprendre le système réactionnaire de l'autoritarisme tsariste, qu'il croyait déjà pareil à la dérive de l'Ancien Régime et à la terreur révolutionnaire. Il s'intéresse surtout aux analogies du système absolutiste tsariste avec la terreur jacobine, narrant souvent en

5. Suivant le nom de l'édition anglaise de 1854-1855.

6. Lilienthal a-t-il lu Custine ? Possible, même très probable, mais cela est assez difficile à prouver, du moins aujourd'hui, dans l'état de son archive. 
menu détail les atrocités et la cruauté du régime policier russe; aussi, son point de vue est celui d'un observateur irrité par la constante hypocrisie du gouvernement russe, cherchant toujours à prouver par ses observations la supériorité de ses opinions politiques sur la monarchie constitutionnelle et sur le républicanisme libéral. C'est dans ce contexte qu'il affiche avec conviction son catholicisme de vocation universaliste: cette idéologie, à la fois religieuse et politique, occupe une position centrale et, somme toute, est établie comme point privilégié d'observation des réalités russes. Considéré comme seule religion universaliste, le catholicisme de Custine se donne comme seul et unique remède aux nationalismes ethniques et religieux centrifuges ${ }^{7}$. À l'aune des renouvellements puissants s'annonçant déjà dans le paysage politique français, la visite russe du marquis de Custine donne un aperçu de l'État politique d'un pays aux limites du désespoir et de la défaite, rongé par les abus en toute sorte, et dont la corruption est générale. Dans cet affreux état de choses, la discrimination religieuse est surtout considérée du point de vue des persécutions réservées aux catholiques russes: «L'Europe occidentale ignore tout ce qu'il entre d'intolérance religieuse dans la politique russe» (1990, vol. 2, 441). D'autre part, dans son analyse, Custine fait aussi voir que, selon son opinion, l'Église orthodoxe russe est réduite au silence et en est venue à partager une complicité malséante avec le système absolutiste. Tout en se demandant à «quoi tient la nullité d'une Église que tout semble favoriser dans son œuvre» (vol. 2, 440), il constate — dans un passage d'analyse très pénétrant: "La foi politique est plus ferme ici que la foi religieuse; l'unité de l'Église grecque n'est qu'apparente: les sectes réduites au silence par le silence habilement calculé de l'Église dominante, creusent leurs chemins sous terre.» (vol. 2, 22)

Max Lilienthal, d'autre part, peut être décrit comme un témoin qui serait l'absolu opposé de Custine: ce jeune rabbin et docteur en philosophie, formé par l'université allemande de son époque dans l'esprit de l'Émancipation juive, part en Russie avec une mission réformatrice et agira, de concert avec Serge Uvarov ${ }^{8}$, dans le but avoué de contribuer à la modernisation et à l'acculturation des Juifs de la Russie, "d'élever leur niveau »! Dans son activité, il se fera l'agent des Lumières juives et, dans ses lettres, il fera part à sa famille de la perte graduelle de ses espoirs et de la nature de ses obstacles.

7. «Partout [...] j'ai senti couver le feu des guerres religieuses» $(1990$, vol 1, 16), et il pense que la solution se trouve dans l'universalisme catholique!

8. Pour l'apport d'Uvarov à la politique réformiste russe sous Nicolas I ${ }^{\text {er }}$, voir Stanislawski 1983 et Whittaker 1978. 
Voyageant en Russie avec le mandat gouvernemental de constater l'état des communautés juives pour ensuite faciliter la mise en place d'un nouveau système administratif et éducatif pour les sujets juifs du Tsar, Max Lilienthal est l'énonciateur d'un fascinant discours sur les Juifs russes, marqué par son utopique départ du réel et du possible. En dépit de ses intentions réformatrices et de son identité juive, il restera un étranger, car il ne connaît aucune des langues parlées par les Juifs russes et polonais : yiddish, hébreu ou russe. En authentique représentant des Lumières occidentales, il entend communiquer partout en allemand et en français! Ainsi, depuis le début, l'espace russe de ses mémoires de voyage s'offre comme territoire de l'étrangeté et de l'incompréhension. Sa place restera ainsi celle d'une liminalité programmatique ${ }^{9}$, se confrontant à la traditionnelle mentalité diasporique juive, à son messianisme et à la redéfinition de ces dimensions dans l'idéologie de l'Émancipation. À mon avis, c'est là où l'intérêt principal de ses lettres est le plus visible, dans la complexité des rôles que le jeune auteur assume et qui s'articulent dans son récit à mesure que sa mission avance... vers l'échec!

Le voyage de Lilienthal à travers l'espace russe de résidence juive est ainsi constitué en espace d'une culture vouée au paradoxe et à des problématisations plurielles ${ }^{10}$. Son récit nous fait connaître le sort d'une minorité jusqu'alors très bien cloisonnée, penchée sur elle-même, résistant par l'isolement à une longue histoire de persécutions.

\section{Les espaces de l'aliénation : territoires juifs et territorialisation moderne}

On sait aujourd'hui que l'isolement juif fut, à travers les siècles, doublement articulé, à la fois imposé de l'extérieur, par force, et construit de l'intérieur, comme moyen de défense et de survie. Mais, si l'histoire millénaire des Juifs prémodernes est celle d'un isolement au cœur des populations de religions différentes, leurs voies parallèles vers une modernité décloisonnante porteront souvent les traces des particularités locales. L'espace de la modernité juive est

9. Je renvoie ici à la définition donnée par V. Turner $(1966,94-96)$ au concept de liminalité, concept qui inclut la condition transitoire, le changement et l'impermanence des rôles sociaux.

10. Dans son analyse du "cas Lilienthal », Michel Meyer (1985) a montré toute la complexité de l'interaction du réformateur allemand avec le complexe et polymorphe espace russe du judaïsme, partagé entre tradition et innovation, dans la première moitié du XIX ${ }^{e}$ siècle. 
ainsi fortement marqué par le passé d'une ségrégation perçue comme volontaire et, de la sorte, on peut dire que le dedans et le dehors de cet isolement se rejoignent pour donner une géographie de ségrégation juive qui reste iconique pour l'histoire intellectuelle moderne des Juifs ${ }^{11}$.

Dans les espaces urbains de l'Europe centrale et occidentale, le quartier juif reçoit, dès le Moyen Âge, un emplacement fermé, le ghetto. En tant que cité juive à l'intérieur de la cité chrétienne homogène qui l'entoure, le ghetto se constitue comme espace liminaire et limité à la fois, qui articule l'identité de ses habitants en vertu d'une différence, perçue en même temps comme religieuse - élaborée en des termes théologiques - et comme mode de vie ${ }^{12}$. Similairement, en terre d'islam, le mellah ${ }^{13}$ — situé en marge de la médina - sépare dans la ville les espaces respectifs des deux religions, la juive et la musulmane (voir Gottreich 2004). De la sorte, la ségrégation spatiale est symbole et icône du contrôle imposé au partage des institutions qui régissent les pratiques religieuses et non religieuses de la vie de tous les jours. C'est cette ségrégation spatiale qui restera pour longtemps la marque visible des limites imposées institutionnellement aux Juifs, de la discrimination générale des Juifs sur la base d'une différence conceptualisée par un discours théologique d'application politique et juridique. L'espace de résidence est normé, réglé et reconnu comme tel. C’est aussi un espace qui rend visible une autorisation identitaire et existentielle : identifiés comme étrangers et minoritaires, ses habitants ne peuvent résider ailleurs, étant souvent empêchés de sortir de leur espace. En revanche, cet espace d'isolement est aussi un espace de protection. Il se pose en outre comme l'espace de la souveraineté juive, car, à l'intérieur de l'espace ainsi délimité, les Juifs jouissent d'une autodétermination qui verra, au fil des années, le développement de systèmes structurés de gouvernement, taxation, arbitrage juridique, éducation, etc.

11. Les conceptualisations structurales de l'espace mémorial suivent généralement les dimensions du symbolique mises en évidence par Maurice Halbwachs dans ses travaux classiques sur la mémoire collective (voir notamment 1968).

12. Dans son ouvrage The Ghetto and the Jews of Rome (s.d.), le grand historien allemand Gregorovius nous a laissé une description célèbre du premier ghetto de Juifs à Rome, institué en 1556 par Paul IV: "Écrasés dans un petit coin déprimant de la ville, de l'autre côté du Tibre, à Trastevere, les Juifs de Rome vivent comme dans l'Antiquité, coupés de l'humanité tout entière " (19).

13. Le mellah est un quartier juif séparé par un mur de la «cité musulmane » marocaine. Les Juifs sont ainsi isolés de la médina réservée à la population arabe de la ville et de la casbah habitée par les élites. Voir à ce sujet l'excellente analyse donnée par Gottreich (2004). 
Dans l'ouest de l'Europe, le ghetto désigne une culture à la fois d'isolement et de cloisonnement, fortement conservatrice et idiosyncrasique, gardant jalousement les assises de son altérité. Avec les Lumières et la propension au progrès, tout cela va changer, les membres réformateurs des communautés juives se donnant comme mandat la sortie du ghetto ${ }^{14}$. C'est une vocation modernisante qui impose avec force la mouvance dans un espace nouveau et étranger, parfois inquiétant, souvent effrayant. L'acculturation juive dans la modernité est ainsi saisie en tant que mouvement territorial concret hors de l'espace emprisonnant du ghetto, comme une déterritorialisation, à la fois concrète et symbolique ${ }^{15}$. Pour la grande majorité des Juifs de l'Europe occidentale et centrale, l'évasion vers l'espace de cette liberté nouvelle a aussi une signification urbaine, car on s'échappe hors d'un quartier circonscrit, vivant selon les prescriptions de la tradition médiévale, vers une ville pleine de promesses.

En Russie, d'autre part, les formes de la ségrégation discriminatoire juive ont reçu une réalisation spatiale différente: par des législations successives, l'administration tsariste a limité le séjour résidentiel et la mouvance des Juifs à un espace circonscrit de l'ouest de l'empire, la Zone ${ }^{16}$ de séjour légal, rompant les barrières urbaines de la "cité juive » médiévale et intégrant les nombreux shtettelach ( «bourgades») dans un réseau discontinu qui transpose les "principes du ghetto » à l'échelle régionale et étatique. En fait, il s'agit d'un redoublement des structures d'isolement, car, à l'intérieur même de la Zone, les Juifs ont la permission de résider seulement dans certaines villes et, à l'intérieur des villes, dans des quartiers circonscrits.

Les limites de l'espace de ségrégation sont à la fois très bien renforcées et tout aussi poreuses, car certaines catégories professionnelles sont autorisées à sortir et même à rester en dehors pour des périodes limitées de séjour prolongé, ainsi changeant leur statut d'étranger (outsider) pour un statut liminaire. Loin d'être homogène, la population juive de l'Empire russe abrite aussi des groupes voués à la mouvance et à la traversée de l'espace. Le symbole matériel palpable de ce statut institutionnel de transition reste, sans doute, le passeport interne.

14. Ou du mellah. Il faut voir dans ce sens l'emblématique choix de résidence dans la médina de M. Montefiore en visite au Maroc (Gottreich 2004, 118-119).

15. Pour une discussion classique de ces aspects, voir Katz 1984.

16. Pale of Settlement, ou cherta [postoyannoy yevreyskoy] osedlosti. 
Les changements réformateurs auront donc pour mission de déconstruire graduellement les limitations juridiques et symboliques de cette régionalisation résidentielle imposée et d'ouvrir les villes du pays à l'immigration interne des Juifs, venant s'installer et s'adapter aux conditions de la vie urbaine moderne ${ }^{17}$.

Dans le récit de Lilienthal, l'histoire des Juifs russes, à l'aune des Lumières doublement importées ${ }^{18}$, reçoit aussi une description spatiale, la narration du voyageur juif déployant la représentation topographique de l'aliénation juive en train de s'ouvrir aux changements modernisateurs. Mais, encore une fois, sa position de cartographe dans la Zone est unique, car il est à la fois membre de cette communauté religieuse des Juifs qu'il veut dépeindre et observateur détaché. Lilienthal exerce donc cette double qualité d'insider et d'outsider partout où il va, mêlant dans ses commentaires également la distance de l'observateur et la bonne volonté de ses intentions et de son optimisme. Et c'est là l'une des qualités de ce texte, fortement controversé: son récit des réalités juives en Russie est d'autant plus intéressant qu'il dresse un tableau à peu près utopique d'un espace d'enquête et de recherche.

Il s'agit d'une géographie symbolique du religieux politisé et de la théologie politique avant la lettre qui confronte en permanence le statu quo avec le changement, donné comme inévitable, et qui prône un progrès à la fois certain et inquiétant.

Une de ces inquiétudes regarde, justement, l'avenir des idées religieuses du judaïsme dans un espace d'acculturation contrôlé par l'État autocrate, dans un contexte qui perçoit déjà la religion comme une dimension à manipuler et à mettre au service du politique. Dans le cas de Lilienthal et de la situation russe, l'axe de partage ne sépare plus le séculier et le sacré, comme dans la pensée de Custine et de Tocqueville ${ }^{19}$, mais il passe entre le conservatisme religieux et le changement "progressif » et rapprochant (acculturant, assimilant à la rigueur) avec les populations avoisinantes.

17. Et aussi, par compensation, chaque fois que le statut des Juifs russes s'aggrave, on assiste à des projets de colonisation par émigration massive dans des territoires perçus comme vierges: l'Algérie française, le Canada, l'Ouganda, la Palestine.

18. Comme on le verra, les modèles allemand et français se confrontent ici, tout en oblitérant un virtuel modèle russe, au demeurant enfoui dans un espace intellectuel méconnu. Voir en ce sens Meyer 1985.

19. À des degrés variés, ils reprennent le mouvement de déchristianisation publique et de sécularisation commencée par la Révolution. 
Comme l'a bien montré Michael Stanislawski dans un ouvrage devenu classique $^{20}$ (1983), le lien idéologique qui réunit Lilienthal, le maskil ${ }^{21}$ allemand, avec les maskilim russes et polonais est celui de la rencontre et du rapprochement, de la compréhension qu'il fallait réduire la distance séparant les Russes et les Juifs. D'autre part, ce qui sépare les représentants russes de la Haskalah et le maskil venu d'ailleurs est le fait que Lilienthal reste programmatiquement un étranger, représentant le pouvoir autocratique du Tsar et du ministre Uvarov, qui, au fait, se servent de lui pour leurs propres fins.

Les voyages de Lilienthal en Russie sont fortement et forcément chargés d'une idéologie réformatrice sans compromis; conséquemment, son récit dévoile des présupposés et des préjugés constamment critiques. En général, on peut dire que les assurances et les incertitudes de Lilienthal sont celles des Lumières, ses malheurs sont en grande partie ceux de l'idéologie du progrès à tout prix, si bien mise en texte dans la littérature française, entre autres par Charles Nodier ${ }^{22}$.

\section{Une topographie de l'isolement et de l'aliénation : les voyages dans la Zone}

Lilienthal a parcouru la Zone par deux fois, ce qui lui a donné l'occasion de déployer une topographie discursive qui reste unique pour l'époque. C'est la topographie du judaïsme russe minoritaire, qui s'étend sur un espace déjà confiné et sanctionné par une politique religieuse des minorités hautement manipulatrice, dissimulant l'obscurantisme et la volonté d'assimilation par conversion au christianisme officiel. Cette politique de conversion au christianisme de rite orthodoxe, intensifiée sous le tsar Nicolas $\mathrm{I}^{\mathrm{er}}$, est vue comme l'élément central d'une politique d'homogénéisation nationale. Chargée de connotations millénaristes, cette intention de conversion au christianisme devient en fait la clef de voûte d'une stratégie "d'amélioration » sociale et culturelle inspirée du projet de "régénération » des Juifs, mis en discours au XVIII ${ }^{\mathrm{e}}$ siècle, débattu par la Convention révolutionnaire et institutionnalisée

20. Certaines des analyses de ce livre ont été revues et raffinées, mais somme toute préservées dans leur ensemble, par des travaux plus récents, comme ceux de I. Bartal (voir 2005) et M. Wodzińsky (voir 2004; 2005).

21. Par maskil (pl. maskilim) on désigne les représentants de la Haskala, c'est-à-dire des Lumières juives.

22. Sa critique de l'idée de progrès est exprimée dans «Le Cycle du Dériseur sensé » (voir Nodier 1961). 
comme programme politique émancipateur par l'État napoléonien. Selon la devise du ministre S.S. Uvarov, "Autocratie, Orthodoxie, Nationalité $(\text { russe })^{23}$ », l'éducation modernisant les Juifs devra aboutir à leur conversion au christianisme orthodoxe pour en faire des meilleurs sujets du Tsar. Dans la vision du ministre Uvarov, l'école est l'institution la plus appropriée pour cette action homogénéisante:

Mon but est de réunir les divers éléments et de les avoir assis sur les bancs d'une seule école. Russes et Polonais, Italiens et Allemands, Français et Moldaves, Juifs, Turcs, Tatars et Arméniens, Grecs et Bulgares. Cela demande, sans doute, beaucoup de travail et du bon sens pour en faire, seulement par éducation, non seulement de bons et utiles citoyens, mais aussi de leur faire comprendre qu'il faut connaître et aimer notre Patrie. (Whittaker 1982, 188)

Mandaté par Uvarov, Lilienthal fera donc deux voyages à travers la Zone - un en 1842 et l'autre en 1843 — afin de communiquer avec les sujets juifs du Tsar, pour, au fait, faire la propagande des idées du ministre Uvarov, dont les véritables intentions lui restent d'ailleurs partiellement cachées. Cela lui donne l'occasion de rencontrer pour la première fois la grande diversité culturelle des communautés juives en Russie. C'est grâce à ces missions commandées qu'a été faite sa description des «espaces juifs» russes.

Sa géographie narrative et religieuse est marquée par les idées préconçues que porte ce «missionnaire de l'émancipation» de modèle allemand. Ainsi, dans son périple à travers les grands espaces enneigés de la Russie juive, Lilienthal privilégie toujours les espaces urbains, s'arrêtant dans les villes qu'il considère comme importantes pour sa mission, mais négligeant toujours les villages, qui, à ce moment-là, étaient le véritable contexte de la vie juive, abritant un mode de vie caractéristique, qui sera de fait identifié avec l'identité particulière du Juif est-européen, l'Ostjüde.

L'espace de l'urbanisme juif qu'il décrit est ainsi un espace représentatif pour ce qu'il pense de la Zone, et il dresse vraiment un tableau pittoresque et perçant de la diversité juive — surtout dans l'espace des pratiques religieuses et éducatives, régies par l'institution de l'autogouvernement juif, le kahal $^{24}$. Mais, comme il va s'en rendre compte lui-même, les différences

23. Donnée comme contrepartie normative au fameux «Libérté, Égalité, Fratérnité » de la Révolution (voir Edwards 1982, 45 ; Whittaker 1984, 94).

24. Perçu comme institution corporative, le kahal fera l'objet des tentatives d'annihilation des ministres tsaristes, qui veulent le remplacer par un consistoire de modèle napoléonien. 
culturelles, surtout religieuses, parmi les Juifs font état d'un pluriculturalisme complexe et compliqué. Après avoir passé la première partie de son séjour en Russie à Riga (Lettonie) et à Mitau (Courlande), parmi des communautés juives germanisées et germanophones, qui lui ont permis d'appliquer plusieurs changements novateurs ${ }^{25}$, Lilienthal se rend à Vilnius (Wilna), en Lituanie, où il sera confronté avec la diversité juive dans toute sa force.

Pour se familiariser un peu avec cette étrangeté, il a recours à une référence napoléonienne: en 1812, entrant à Vilnius, l'Empereur se serait exclamé: «Messieurs, je crois que nous sommes ici à Jérusalem. »Et à Lilienthal d'ajouter: "Moi aussi je me croyais en Palestine et non en Russie, tellement la ville était remplie de Juifs» (Philipson 1915, 261). Surpris, émerveillé, il décrit avec animation un grand espace urbain, entièrement juif, avec des institutions juives et une architecture urbaine et résidentielle juive. Habitué aux petits espaces des ghettos allemands, la vue d'un espace urbain de prédominance juive lui semble exceptionnelle et il ne finit pas de l'évoquer, souvent avec humour: "Vilnius, à l'époque, comptait trente mille Juifs et il me semble que trois quarts d'entre eux sont toujours dans la rue, chassant un emploi. Toutes les boutiques appartiennent aux Juifs; les femmes s'en occupent et, avec leurs yeux noirs, guettent continûment les clients. Les hommes sont surtout engagés dans des affaires à l'extérieur » (261-262). Cet espace du commerce et du métier juifs est construit aussi comme espace du regard ethnographique, par des descriptions détaillées des costumes et coutumes divers. Finalement, l'espace de la ville est articulé discursivement comme espace de la religion et de l'instruction dans les quelque 40 pages qui suivent (260-300).

Si Vilnius est l'espace d'une urbanité juive en pleine effervescence, surprise à l'heure du changement, où la tradition et l'innovation se confrontent avec force, Minsk est la ville où la tradition est maintenue avec un conservatisme outrancier et figé : "Minsk en 1841 était un des piliers du rabbinisme russe » (305). Là, Lilienthal décrit un contexte dans lequel règne une sorte de dogmatisme religieux rigoriste, fait d'instruction en Talmud et de pilpul (casuistique talmudique, souvent excessivement élaborée). Les représentants de ce conservatisme religieux, les mitnaggedim, sont seulement contrariés par l'arrivée de l'hassidisme (piétisme juif). Mais, comme le remarque Lilienthal, les deux orientations, l'une dominée par un rationalisme excessif, l'autre,

25. Sur les plans de l'éducation, de l'habillement et de la liturgie. 
par l'émotivité, se rencontrent pour s'opposer à la réforme et à l'innovation. Dans ces conditions, le centre de la ville juive reste indiscutablement la synagogue. Minsk représente la ville de l'adversité à la réforme et est décrite comme le siège central d'une opposition acharnée à la modernité, où le conservatisme se fait violent ${ }^{26}$. Menacé, Lilienthal sera forcé de fuir la ville de Minsk.

De retour sain et sauf à Vilnius, il aura à faire face à une résistance de nature différente. C'est une opposition à ses propos qui est beaucoup mieux articulée, car elle énonce la peur du pouvoir autoritaire et met en lumière l'insuffisance des projets réformateurs de modèle franco-allemand dans le contexte russe et polonais.

Le périple de Lilienthal dans la Russie des Juifs continuera par des visites à Grodno, Jitomir, Berdichev, Kaments-Podolsk, Chisinau, Odessa, Kiev, Cernigov, Mogilev et Vitebsk. Seulement une partie de ces importants centres de la vie juive sous Nicolas ${ }^{\text {er }}$ ont reçu une description dans ses lettres. C'est que son voyage à travers la Zone est de plus en plus senti comme une mission policière. Son service commandé est de toute évidence - comme dans le cas de Custine - sujet aux pressions et à la surveillance du régime tsariste. Dans l'absence des droits civils octroyés aux Juifs, leur « régénération " perd son sens émancipateur. Revenu de son voyage à travers la Russie juive, averti par sa famille qu'il risque de se faire l'outil d'une oppression encore plus intense, Lilienthal mettra toute son inventivité dans l'effort d'échapper du panopticon russe.

L'espace des voyages de Lilienthal en Russie est celui du religieux à l'aune des changements imminents, qui ont pour but une intégration forcée et unilatérale dans un territoire jusqu'alors interdit. Mais comme les objectifs du gouvernement tsariste sont trompeurs, l'accès aux droits civils l'arrière-fond d'un système social libéralisé —, qui aurait encouragé enfin la sortie de la Zone, est refusé aux Juifs. Au but de son périple russe, Max Lilienthal sera en fait arrivé à la même conclusion que le marquis de Custine: la Russie est le territoire du mensonge officiel, les intentions réformatrices du gouvernement ne font qu'empirer la crise des minorités visées.

Si Custine était revenu en France avec une foi nouvelle dans l'avenir du système représentatif (français) et avec la conviction que « [1]'âge politique du christianisme finit» (selon le mot de Chateaubriand en 1831), Lilienthal,

26. Exprimé dans un langage russe-yiddish, de mélange significatif et de sonorités comiques: shkoles ne jelaiem, "on veut pas d'écoles!" (Stanislawski 1983, 74). 
lui, prendra encore quelque temps pour se rendre compte de la situation réelle et décider de son avenir. Encore une fois, il "votera avec ses pieds». En 1845, Lilienthal quitte la Russie et part pour l'Amérique ou il deviendra un des représentants importants et influents du mouvement d'Émancipation juive aux États-Unis (voir Temkin 1976; Ruben 1997; 2003).

L'expérience russe lui aura aussi servi à comprendre les limites des innovations religieuses et il montrera beaucoup plus de prudence et de prévoyance dans sa carrière de réformateur religieux chez les Américains. En même temps, il gardera toujours une volonté vive de rapprochement avec des groupes de foi différente de la sienne. Grâce à cette attitude humaniste et tolérante, à la fois flexible et active, il deviendra l'un des «inventeurs » de la prédication œcuménique, l'apôtre juif d'une foi nouvelle dans la coexistence pacifique.

\section{Références}

Ages, A. (1970), French Enlightenment and Rabbinic Tradition, Francfortsur-le-Main, Klostermann.

Baron, S.W. (1987), The Russian Jew under Tsars and Soviets, New York, Shocken Books.

BARTAL, I. (2005) [hébreu 2002], The Jews of Eastern Europe, 1772-1881 / trad. par C. Naor, Philadelphie, Penn Press.

Birnbaum, P. et I. KatZnelson, dir., (1995), Paths of Emancipation. Jews, State and Citizenship, Princeton, Princeton University Press.

Cohen, I. (1943), Vilna, Philadelphie, JPSA (Jewish Communities Series).

Custine, A. de (1838), L'Espagne sous Ferdinand VII, Paris, Ladvocat, 4 t. reliés en 2 vol. (1990) [1843], La Russie en 1839, Paris, Solin, 2 vol.

Dubnow, S. (1918), History of the Jews in Russia and Poland, Philadelphie, JPSA.

Edwards, D.E. (1982), «Nicholas I and Jewish Education », History of Education Quarterly, 22/1, p. 45-53.

FeINer, S. (2004) [hébreu 2002], The Jewish Enlightenment / trad. par C. Naor, Philadelphie, Philadelphia University Press.

FRIEDLÄNDER, D. (1819), Ueber die Verbesserung der Israeliten in Königreich Pohlen, Berlin, Nicolai. 
Gardet, L. (1956), La cité musulmane. Vie sociale et politique, Paris, Vrin (Études musulmanes).

Ginsburg, S.M. (1939), «Max Lilienthal's Activities in Russia. New Documents ", Publications of American Jewish Historical Society, 35, p. 39-51.

GotTreich, E. (2004), «Rethinking the «Islamic City» from the Perspective of Jewish Space », Jewish Social Studies, 11/1, p. 118-146.

Greenberg, L. (1965), The Jews in Russia. The Struggle for Emancipation, Yale, Yale University Press.

Gregorovius, F. (s.d.), The Ghetto and the Jews of Rome, New York, Shocken Books.

Halbwachs, M. (1968), La mémoire collective, Paris, Presses universitaires de France.

Hobswam, E. (2005), «Benefits of Diaspora ", London Review of Books, 27/20, p. 25.

KASPI, A., dir. (1989), La Révolution française et l'émancipation des Juifs de France, Paris, Hamore.

KaTZ, J. (1984), Hors du ghetto. L'émancipation des Juifs en Europe (17701870), Paris, Hachette.

— dir. (1987), Toward Modernity. The European Jewish Model, New Brunswick / Oxford, Transaction Books.

Kennan, G.F. (1971), The Marquis de Custine and His Russia in 1839, Princeton, Princeton University Press.

KLIER, J.D. (1986), Russia Gathers Her Jews. The Origin of the "Jewish Question» in Russia, 1772-1825, DeKalb, Northern Illinois University Press.

Lebrun, F., dir. (1980), Histoire des catholiques en France, Paris, Privat (Pluriel).

Leskov, N. (1986), The Jews in Russia. Some Notes on the Jewish Question / édité et traduit par H.K. Schefski, Princeton, Princeton University Press.

Mendes-Flohr, P. et Y. ReInharz (1980), The Jew in the Modern World. A Documentary History, Oxford / New York, Oxford University Press.

Meyer, M. (1985), "The German Model of Religious Reform and Russian Jewry ", dans I. TWERSKY, dir. (1985), Danzig, Between East and West: Aspects of Modern Jewish History, Cambridge, Harvard University Press, p. 65-91. 
Nodier, C. (1961), Contes / texte établi par P.G. Castex, Paris, Garnier.

Philipson, D. (1915), Max Lilienthal, American Rabbi: Life and Writings, New York, Bloch.

RaIsIN, J. (1913), The Haskalah Movement in Russia, Philadelphie, JPSA.

Ruben, B.L. (1997), Max Lilienthal: Rabbi, Educator and Reformer in Nineteenth Century America, thèse de doctorat (non publiée), City University of New York.

(2003), « Max Lilienthal and Isaac M. Wise: Architects of American Reform Judaism », The American Jewish Archives Journal, 55/2, p. 1-29.

Schorsch, I. (1994), From Text to Context. The Turn to History in Modern Judaism, Hanover, Brandeis University Press (Tauber Institute for the Study of European Jewry Series).

Stanislawski, M. (1983), Tsar Nicholas I and the Jews, Philadelphie, JPSA.

Temkin, S.D. (1976), "Rabbi Max Lilienthal Views American Jewry in 1847 », dans B.W. KoRN, dir., A Bicentennial Festschrift for Jacob Rader Marcus, New York, Ktav Publishing House, p. 589-608.

Tocqueville, A. de (1835), De la Démocratie en Amérique, Paris, Charles Gosselin.

Tollet, D. (1992), Histoire des Juifs en Pologne, Paris, Presses universitaires de France.

Turner, V. (1966), The Ritual Process. Structure and Anti-Structure, Ithaca / New York, Cornell University Press.

WhitTAKeR, C.H. (1978), «The Ideology of Sergei Uvarov: An Interpretive Essay », Russian Review, 37/2, p. 158-176.

(1984), The Origins of Modern Russian Education: An Intellectual Biography of Count Sergei Uvarov, 1786-1855, DeKalb, Northern Illinois University Press.

WodZIŃski, M. (2004), "Good Maskilim and Bad Assimilationists, or Toward a New Historiography of Haskalah in Poland ", Jewish Social Studies, 10/3, p. 87-122.

(2005), Haskalah and Hasidism in the Kingdom of Poland. A History of Conflict, Oxford / Portland / Oregon, Littman Library of Jewish Civilization. 


\section{Résumé}

L'article examine les mémoires des voyages en Russie de Max Lilienthal, un texte d'exception sur la Russie juive: le jeune voyageur juif venu d'Allemagne décrit l'image d'une communauté religieuse et ethnique à l'aune de la crise dramatique des Lumières, drame qui se déploie sur le territoire de la Russie tsariste. À la fois engagé et curieux de tout ce qui a trait à la situation des Juifs, Max Lilienthal construit dans ses lettres de Russie un espace idéologique particulier, à la fois cloisonné et divers, cartographiant la condition juive dans l'empire de Nicolas I $^{\mathrm{er}}$ et imposant une comparaison éclairante avec le texte du voyage russe d'Astolphe de Custine, écrit à la même époque, mais beaucoup mieux connu.

\section{Abstract}

This paper examines the image of the Russian Jewry in the memoir of Max Lilienthal, a Jewish-German author who explored the Russian Pale of settlement on official mandate in 1839. In so doing, he built a representational space of a persecuted minority in the tsarist empire, a minority already diversified and fragmented, under complex and polarizing pressures to reform (within traditional frames). The many challenges of Jewish modernization under Nicholas I, as understood by Lilienthal, become even more dramatic when compared with a contemporary text of travels in the Russian empire, the famous "La Russie en 1839 » by Astolphe de Custine, a text that gave an iconic representation of the police state in its dealings with minority religious and ethnic groups. 